\title{
CONVECTIVE ZONES IN THE ENVELOPE OF MASSIVE
}

\section{STARS}

\author{
FRANK M. ALBERTS \\ Astronomical Institute 'Anton Pannekoek' and Center for High Energy Astrophysics \\ University of Amsterdam, Kruislaan 403, 1098 SJ Amsterdam, Netherlands
}

In the calculation of stellar models with the Cox-Stewart opacities no convective zones in the outer layers of massive stars appear. The new OPAL opacities (Rogers \& Iglesias, 1992) show a significant bump in the opacity near temperatures of $\log T=5.2$. This opacity effect results in a small convective zone in the envelope of stars with mass ranging from $15 \mathrm{M}_{\odot}$ to $150 \mathrm{M}_{\odot}$, apart from possible convective zones caused by ionization. This was also briefly mentioned by Glatzel \& Kiriakidis (1993). For stars on the main sequence this zone is small, about $1 \%$ of its radius on the zero age main sequence up to $7 \%$ at the onset of the core helium burning and contains a negligible amount of mass. For helium burning stars, however, this convective zone moves inward, keeping the same size but containing more and more mass.

The effect of the opacity on the quantity $\nabla_{\mathrm{rad}}-\nabla_{\mathrm{ad}}$ is shown in the figures. A contour is plotted where this quantity equals zero. The outlined regions are therefore convective zones by the Schwarzschild criterion. Figure 1 shows the convective regions for a $20 M_{\odot} \operatorname{star}(\mathrm{X}=0.70, \mathrm{Z}=0.02)$ as a function of time and radius. Figure 2 shows the convective regions for ZAMS stars as a function of mass in the range between $1 M_{\odot}$ and $145 M_{\odot}$. Only the outer parts of the star $\left(0.9 R_{\star}\right.$ to $\left.1.0 R_{\star}\right)$ are shown.

The models have been calculated with the stellar evolution code developed by Eggleton (1971, private communication) in which semi-convection is treated as a slow diffusion process. Mass loss and convective overshooting have not been taken into account.

Consequences of the effects of the presence of this convective zone for oscillatory motion in these stars are currently being investigated.

\section{References}

Eggleton P.P., 1971, MNRAS, 151, 351

Glatzel W. and Kiriakidis M., 1993, MNRAS, 262, 85

Rogers F.J. and Iglesias C.A., 1992, ApJS, 79, 507 

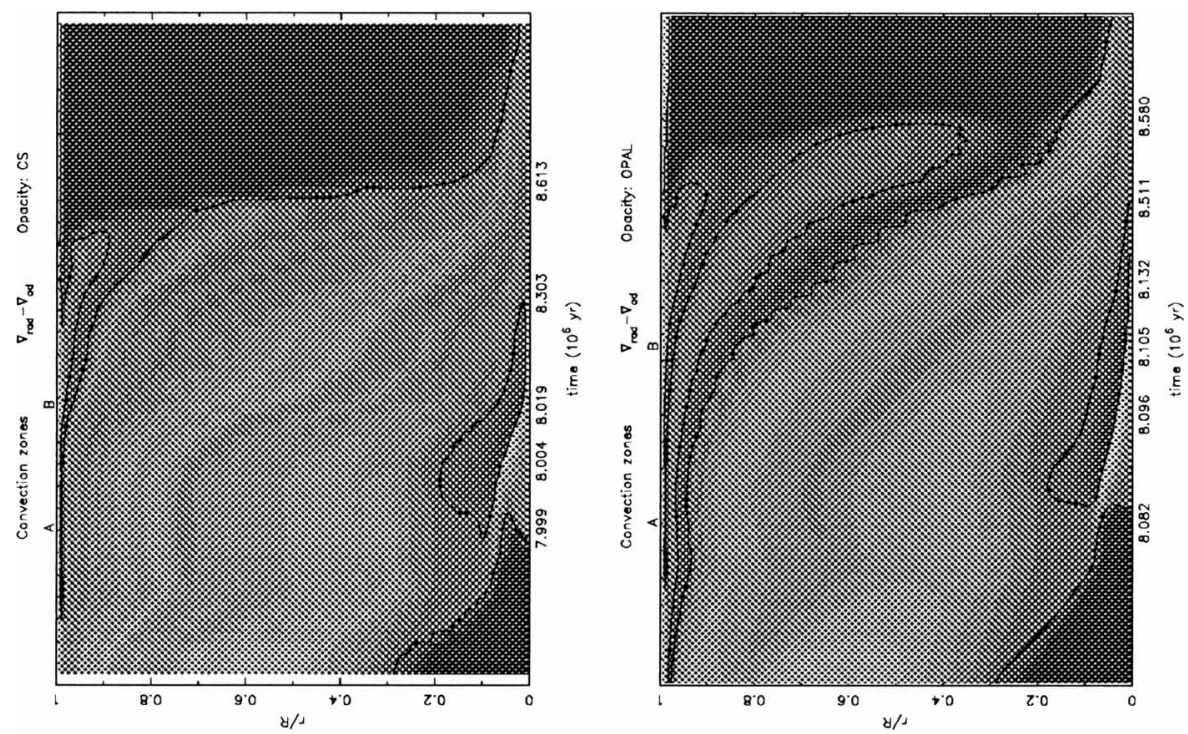

Fig. 1. Convective regions for a $20 \mathrm{M}_{\odot}$ star with the Cox-Stewart opacities (left) and the OPAL opacities (right). The $x$-axis displays the evolution in time. The letters at the top of the plot indicate the evolution phase: A - End of hydrogen core burning, B - Start of helium core burning. The dark shading corresponds to convective regions. Note that the horizontal scale is not linear
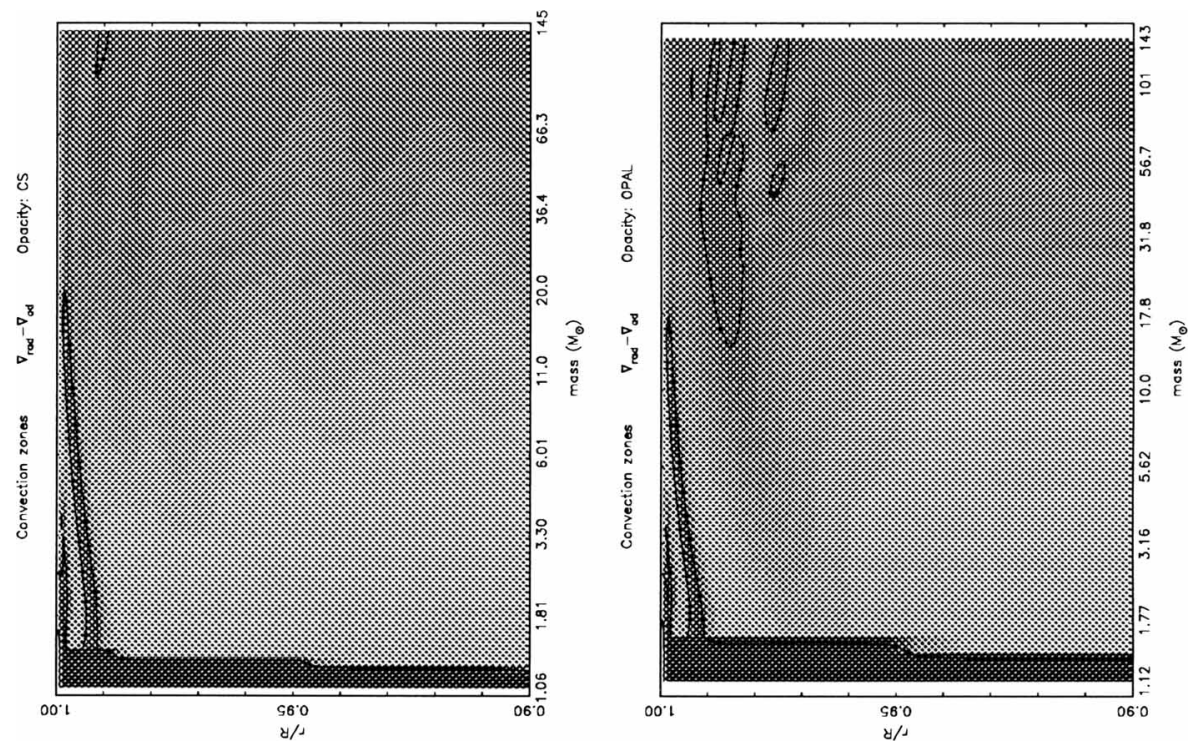

Fig. 2. Convective regions for ZAMS stars, calculated with the Cox-Stewart opacities (left) and the OPAL opacities (right). Note that the horizontal scale is not linear. 